Molecular Breeding 1: 303, 1995.

Erratum

\title{
Genetic engineering of reproductive sterility in forest trees
}

Steven H. Strauss*, William H. Rottmann, Amy M. Brunner and Lorraine A. Sheppard Department of Forest Science, Oregon State University, Corvallis, OR 97331-7501, USA (*author for correspondence; fax: 503 737-1393)

Due to an error, the fax number mentioned in the affiliation was wrong. It should be: $+1-503-737-1393$. 